\title{
The "edge effect" phenomenon: deriving population abundance patterns from individual animal movement decisions
}

\author{
Jonathan R. Potts ${ }^{1}$. Thomas Hillen ${ }^{2}$ Mark A. Lewis ${ }^{2,3}$
}

Received: 16 June 2015 / Accepted: 22 October 2015 / Published online: 23 December 2015

(C) The Author(s) 2015. This article is published with open access at Springerlink.com

\begin{abstract}
Edge effects have been observed in a vast spectrum of animal populations. They occur where two conjoining habitats interact to create ecological phenomena that are not present in either habitat separately. On the individuallevel, an edge effect is a change in behavioral tendency on or near the edge. On the population-level, it is a pattern of population abundance near an edge that cannot be explained in terms of either habitat in isolation. That these two levels of description exist suggests there ought to be a mathematical link between them. Here, we make inroads into providing such a link, deriving analytic expressions describing oftobserved population abundance patterns from a model of movement decisions near edges. Depending on the model parameters, we can see positive, negative, or transitional edge effects emerge. Importantly, the distance over which animals make their decisions to move between habitats turns out to be a key factor in quantifying the magnitude of certain observed edge effects.
\end{abstract}

Jonathan R. Potts

j.potts@sheffield.ac.uk

Thomas Hillen

thillen@ualberta.ca

Mark A. Lewis

mark.lewis@ualberta.ca

1 School of Mathematics and Statistics, University of Sheffield, Hicks Building, Hounsfield Road, Sheffield S3 7RH, UK

2 Centre for Mathematical Biology, Department of Mathematical and Statistical Sciences, $632 \mathrm{CAB}$, University of Alberta, Edmonton T6G 2G1, Canada

3 Department of Biological Sciences, University of Alberta, Edmonton, Canada
Keywords Advection-diffusion · Animal movement · Edge effect - Landscape complexity - Mathematical ecology · Partial differential equations - Step selection .

Transport equations

\section{Introduction}

Edges separating habitats affect the behavior and demographics of animal populations across a wide range of taxa, from fish to birds, from insects to mammals [e.g., Lidicker and William (1999), Laurance et al. (2004), Batáry et al. (2009), and Macreadie et al. (2010)]. These so-called "edge effects" are multi-faceted and depend on the nature of the edge itself, the habitats on either side, and the behavioral tendencies of the animals (Ries et al. 2004). However, there is a common feature: that the edge between two habitats can affect animal behavior in ways that cannot be explained in terms of either habitat in isolation.

The implication of this phenomenon is that ecosystem services provided by a landscape cannot be understood purely by assessing (i) how much of each type of habitat is present in the landscape, and (ii) which ecosystem services each habitat provides. Rather, the interaction between habitats, as well as the geometric details of how they tessellate the landscape, can have a large effect. Moreover, the effects can be complex and difficult to unravel. For example, the question "in which situations it is better to build a Single Large conservation area Or Several Small ones?" is the subject of ongoing debate (the SLOSS debate), despite many decades of research. Much of the debate boils down to understanding the myriad possible effects of edges of the conservation areas on the animals living there (Burkey 
1989; McNeill and Fairweather 1993; Salomon et al. 2002; Tjrve 2010).

As well as these concerns, understanding how and why edge effects emerge is vital for accurately estimating population demographics. Resource selection analysis (Manly et al. 2002), though very widely used, traditionally assumes that population abundance is a function of the underlying habitat quality. On the other hand, an edge effect means that population abundance in a given habitat is different near the edge to within the middle of a large patch of the same habitat-type. Some recent studies (Barnett and Moorcroft 2008; Potts et al. 2014a) point to the idea that this contradiction might be resolved by incorporating movement into the resource selection procedure. After all, animals are not able to assess the whole landscape before deciding where to reside, as they are limited by their ability to move and to perceive the surrounding landscape. Therefore, the distance over which animals are making their decisions to move, as well as the frequency of those decisions, will likely have a big effect on the locations at which they tend to be observed.

Indeed, the phrase "edge effect" is used interchangeably to refer to individual-level movement decisions and population-level patterns. On the individual-level, an edge effect means a change in an animal's movement patterns near an edge (Laurance et al. 2004; Schultz et al. 2012). For example, this can be a propensity either to cross edges-a positive edge effect, or avoid them-a negative edge effect. On the population level, a negative (resp. positive) edge effect is reported if the population abundance is lower (resp. higher) than average near an edge (Laurance et al. 2004; Batáry et al. 2009). There are also transitional edge effects, where a sharp edge between habitats causes a much more gradual transition in population abundance [e.g. Lidicker and William (1999)]. This conflation of language suggests that individual-level decisions about how to move near edges, taking place on a relatively small spatio-temporal scale, ought to be responsible for patterns of abundance observed on a much larger scale. If so, it should be possible to derive mathematically the various population-level patterns typically observed at edges from rules describing the underlying movement decisions of individual animals. The aim of this paper is to make inroads into providing such mathematical analysis.

We set up a stochastic model of individual movement decisions near a habitat edge that explicitly incorporates the frequency those decisions-i.e., how far into the future is an animal looking as it makes its decision to move in a particular direction. We use this model to derive a partial differential equation (PDE) describing the population abundance distribution that arises from many animals moving according to such movement rules. The steady-state of the PDE is solved exactly to give an analytic expression of the predicted population abundance patterns. Depending on the input parameters, we observe either positive, negative, or transitional edge effects. Importantly, the distance an animal is likely to move between successive habitat-selection decisions is key to the scale of these patterns. We compare our results to two other approaches to deriving PDEs from individual-level decisions.

We focus on edge effects caused by habitat selection decisions made as the animal moves. The model uses a simple landscape consisting of two distinct habitats, with the "edge" between them being implicitly defined (Fig. 1). Sometimes, edge effects occur because the edge provides a unique habitat in and of itself. In such cases, the correct model would have at least three habitats, with a thin "edge habitat" squeezed between the other two. Indeed, more complicated models could incorporate larger numbers of habitats in a variety of geometric configurations. While our modeling framework can be readily extended to explore these scenarios numerically [see, e.g., Potts et al. (2014a)], we focus here on a case simple enough to gain clear analytic insight.

The paper is organized as follows. The "Modelling movement near edges" section sets up the movement model. "A partial differential equation approximation" section derives the population abundance patterns. "Comparison with other

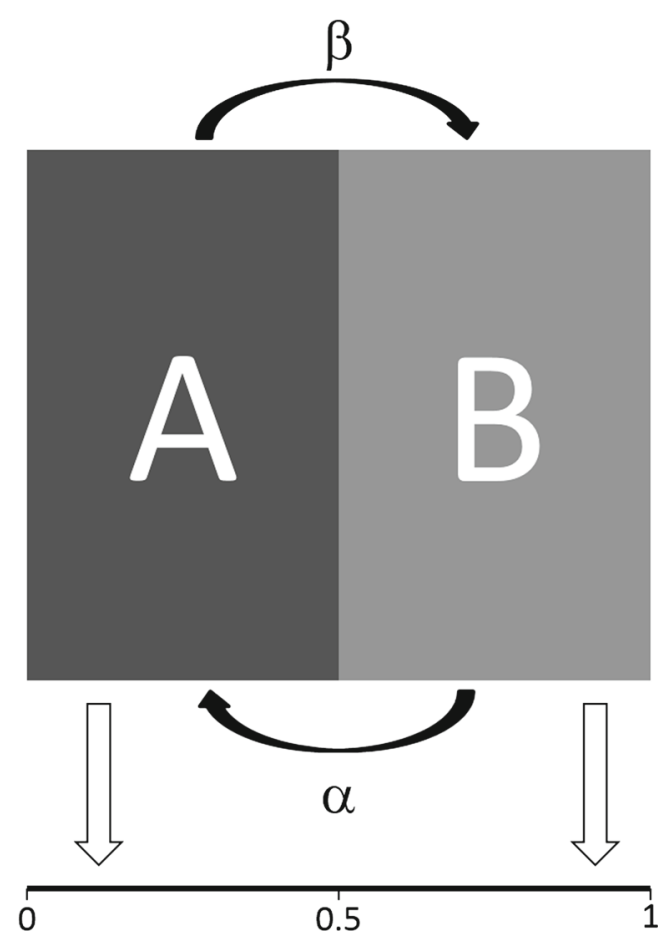

Fig. 1 Model habitat. To model a habitat edge, we use a simple environment with two habitats, $\mathrm{A}$ and $\mathrm{B}$. The weighting for moving from $\mathrm{A}$ to $\mathrm{B}$ is given by $\beta$, whereas $\alpha$ denotes the weighting for moving from $\mathrm{B}$ to $\mathrm{A}$ 
approaches" section examines other approaches to deriving abundance patterns, comparing them to ours. Discussion and concluding remarks are given in "Discussion and conclusions" section.

\section{Modelling movement near edges}

\section{The general movement kernel framework}

The model is based on a discrete-time movement kernel $k_{\tau}\left(z \mid x, \theta_{0}, E\right)$, which describes the probability of an animal moving through an environment $E$ to position $z$ at a time $\tau$ in the future, given that it is currently at a position $x$ and has arrived there on a bearing $\theta_{0}$. These often appear in the ecological literature as step selection functions and can be readily fitted to movement data using techniques that are now relatively standard (e.g., Fortin et al. 2005; Forester et al. 2009; Merkle et al. 2014; Thurfjell et al. 2014). They enable the use of every location in a data set, together with environmental information, to uncover the effects of landscape covariates on animal movement. Therefore, it is sensible to begin with the description of a movement kernel so that our theory fits in with the movement models that are typically used by landscape ecologists.

Here, we consider a form of movement kernel that contains a random element to model the unknown drivers behind movement, together with a weighting function that models the effect of the environment on movement. The random element is a probability distribution, $\phi_{\tau}\left(z \mid x, \theta_{0}\right)$, of possible positions after moving for a time $\tau$, disregarding environmental effects. The weighting function for an animal moving through a static, heterogeneous environment can be written as $W[H(z), H(x)]$, where $H(x)$ is the habitat type at $x$, where the animal currently resides, and $H(z)$ is the habitat at $z$, where the animal is choosing to move. As such, we arrive at the following formula for the movement kernel (Potts et al. 2014a) (henceforth dropping the explicit dependence on the environment, $E$, as we assume this remains constant in time)

$k_{\tau}\left(z \mid x, \theta_{0}\right)=g(x)^{-1} \phi_{\tau}\left(z \mid x, \theta_{0}\right) W[H(z), H(x)]$,

where $g(x)$ is a normalizing function

$g(x)=\int_{\Omega} \phi_{\tau}\left(z \mid x, \theta_{0}\right) W[H(z), H(x)] \mathrm{d} z$,

and $\Omega$ is the study area, so that $k_{\tau}\left(z \mid x, \theta_{0}\right)$ is a probability distribution. Though we separate out the resource weighting function $W$ from probability distribution $\phi_{\tau}$, which contains information about the distance an animal tends to move in a time-step $\tau$, the two aspects of the movement kernel are intrinsically linked. Indeed, one should pick $\tau$ to reflect the frequency with which the animal makes a decision regarding which habitats to move from and to. Consequently, given the speed at which the animal moves, the standard deviation of $\phi_{\tau}$ reflects the spatial scale over which such a decision is made.

\section{A model of movement near edges}

To gain analytic insight into how the scale of behavioral decisions affect the population-level patterns near edges, we examine a particular one dimensional (1D) version of Eq. 1 . For simplicity, we assume that the resource independent movement kernel $\phi_{\tau}\left(z \mid x, \theta_{0}\right)=\rho_{\tau}(z-x)$ is only dependent on the difference $z-x$. Furthermore, we assume that $\rho_{\tau}(r)$ is normally distributed with mean 0 and standard deviation $\sigma$.

The landscape is modeled as a unit interval consisting of two habitats, A and B. Habitat A consists of the left-hand half of the interval, $[0,1 / 2]$, and habitat $B$ consists of the right-hand half, $(1 / 2,1]$, so that $H(x)=A$ for $x \in[0,1 / 2]$ and $H(x)=B$ for $x \in(1 / 2,1]$. The weighting function $W[H(z), H(x)]$ is given by $W[A, A]=W[B, B]=$ 1 (the weighting given for staying in the same habitat), $W[A, B]=\alpha$ (the weighting given for moving from $\mathrm{B}$ to $\mathrm{A}$ ), and $W[B, A]=\beta$ (the weighting given for moving from A to $B)$. In summary, the movement kernel for this model is as follows

$k_{\tau}(z \mid x)=\frac{\exp \left[-(z-x)^{2} / 2 \sigma^{2}\right] W[H(z), H(x)]}{g(x) \sigma \sqrt{2 \pi}}$,

where $g(x)$ is a normalizing function ensuring that $\int_{0}^{1} k_{\tau}(z \mid x) \mathrm{d} z=1$ for each $x \in[0,1]$. Figure 1 gives a picture of this situation, demonstrating how this can be viewed as a projection of a more realistic $2 \mathrm{D}$ scenario.

\section{A partial differential equation approximation}

To derive the population abundance patterns that emerge from our movement model, we use a partial differential equation (PDE) formalism. We make use of recent developments in the theory of transport equations (TEs), introduced by Othmer et al. (1988) and recently reviewed by Hillen and Painter (2013). We refer the reader to those papers to obtain a full description of the underlying mathematical framework, rather than repeating it here. Instead, we go through the details in a brisk fashion, for the purposes of introducing notation and relating TE theory to the movement kernel formalism.

The key ingredient for our TE is the turning kernel, which gives the probability $\mathcal{T}_{\mu}\left(x, v, v_{0}\right)$ of an animal at position $x$ and moving with velocity $v_{0}$, turning to move with velocity $v$, given that it makes a turn. Turns are made at a rate $\mu$. The turning kernel is not precisely equivalent to the 
movement kernel (1); however, we can translate between the two formalisms using the following ansatz

$\mathcal{T}_{2 / \tau}\left(x, v, v_{0}\right)=\tau k_{\tau}\left(x+\tau v \mid x, \theta_{v_{0}}\right)$,

where $\theta_{v_{0}}$ is the direction of $v_{0}$. The intuition behind this ansatz is that the rate of turning in the turning kernel description (left-hand side) should be inversely proportional to the length of a time-step in the movement kernel description (right-hand side), i.e., $\mu \propto 1 / \tau$. That the constant of proportionality is 2 is justified later, when we derive drift terms in PDE expressions both from the turning kernel $(10,18)$ and the movement kernel $(21,22)$ and notice that they are equal. Were $\mu$ set to be $a / \tau$ for some $a \neq 2$ then these drift terms would not coincide, but would differ by a factor of $a / 2$.

The turning kernel feeds into an integro-differential equation describing the probability $p(t, x, v)$ of an animal having particular space-time-velocity co-ordinates (Hillen 2006). For the purposes of this paper, we call this the transport equation (TE), given in $1 \mathrm{D}$ as follows (Othmer et al. 1988)

$$
\begin{aligned}
& \frac{\partial p}{\partial t}(t, x, v)+v \frac{\partial}{\partial x} p(t, x, v) \\
& =\mu \int_{V} \mathcal{T}_{\mu}\left(x, v, v_{0}\right) p\left(t, x, v_{0}\right) \mathrm{d} v_{0}-\mu p(t, x, v),
\end{aligned}
$$

where $p(t, x, v)$ is the probability of being at position $x$ at time $t$, moving with velocity $v$, and $V$ is the space of possible velocities for the animal. Note that Eq. 5 can be generalized to arbitrary dimensions if required (Othmer et al. 1988).

For our model of movement near edges (3), the turning kernel can be written as

$$
\begin{aligned}
q(x, v) & =\mathcal{T}_{2 / \tau}\left(x, v, v_{0}\right) \\
& =\frac{\rho_{\tau}(\tau|v|) W[H(x+\tau v), H(x)]}{\int_{V} \rho_{\tau}(\tau|v|) W[H(x+\tau v), H(x)] \mathrm{d} v},
\end{aligned}
$$

which is no longer dependent upon the velocity $v_{0}$ at which the animal is travelling before it turns.

By writing $u(x, t)$ for the positional probability density, and plugging Eq. 6 into the general equation for the TE (5), we obtain the following integro-differential equation describing the evolution of $p(t, x, v)$

$$
\begin{aligned}
\frac{\partial p}{\partial t}(t, x, v)+v \frac{\partial}{\partial x} p(t, x, v) & =\mu[u(x, t) q(x, v) \\
& -p(t, x, v)], \\
u(x, t) & =\int_{V} p(t, x, v) \mathrm{d} v .
\end{aligned}
$$

To turn the integro-PDE of Eq. 7 into an approximate PDE in $u(x, t)$, there are at least the following three strategies: parabolic scaling, hyperbolic scaling, and moment closure (Hillen 2006; Hillen and Painter 2013). The parabolic scaling technique requires that the expectation of the velocity, $c(x)$, is small enough to be neglected. This turns out not to be the case for our model (see Eq. 15 below), so this scaling method is not appropriate for our study.

The hyperbolic scaling, on the other hand, does not impose such requirements on $c(x)$. However, as explained in (Hillen and Painter 2013), the mathematical theory requires that we pick a small number $\epsilon>0$ such that

$\vartheta=\epsilon t, \quad \xi=\epsilon x$.

If this rescaling were applied to data, it would be necessary to ensure that $\vartheta$ has units such that the time scale over which the data were gathered is roughly $\vartheta=1$, and $\xi$ has units such that the spatial scale over which the data were gathered is roughly $\xi=1$. Notice that for this to be true, the mean speed has to be of order 1 in the units chosen.

To give a biological example to motivate this choice of units, consider the study of caribou movement from Potts et al. (2014a). These data were taken over a 123-day calving season, from 1 May to 1 September. Each caribou herd ranged over an area whose width was up to $100 \mathrm{~km}$. Consecutive position measurements were taken at a resolution of $2 \mathrm{~h}$ and each caribou moved around $0.2 \mathrm{~km}$ between 2$\mathrm{h}$ fixes, so one unit of $t$ is $2 \mathrm{~h}$ and one unit of $x$ is $0.2 \mathrm{~km}$. In this case, we might take $\epsilon=10^{-3}$, so that 1 unit of $\vartheta$ is $2000 \mathrm{~h}$ and 1 unit of $\xi$ is $200 \mathrm{~km}$. Then the calving season is $\vartheta=1.476$ and the home range width is roughly $\xi=0.5$, both of order 1 in the chosen units.

Using this hyperbolic scaling and returning to the original variables (see Appendix A for details), we obtain the following PDE in $(x, t)$ coordinates

$$
\begin{aligned}
\epsilon^{2} \frac{\partial u}{\partial t}(x, t)= & \frac{\tau}{2} \frac{\partial^{2}}{\partial x^{2}}[D(x) u(x, t)]-\frac{\partial}{\partial x}[c(x) u(x, t)] \\
& +\frac{\tau}{2} \frac{\partial}{\partial x}\left[c(x) \frac{\partial c(x)}{\partial x} u(x, t)\right]
\end{aligned}
$$

where

$c(x)=\frac{1}{\tau} \int_{0}^{1}(z-x) k_{\tau}(z \mid x) \mathrm{d} z$,

and

$D(x)=\frac{1}{\tau^{2}} \int_{0}^{1}(z-x)^{2} k_{\tau}(z \mid x) \mathrm{d} z-c(x)^{2}$.

We also impose the following zero-flux boundary conditions

$\left.\left\{\frac{\tau}{2} \frac{\partial}{\partial x}[D(x) u(x, t)]-[c(x) u(x, t)]+\frac{\tau}{2}\left[c(x) \frac{\partial c}{\partial x} u(x, t)\right]\right\}\right|_{x=0,1}=0$.

We are interested in calculating long-term population abundance. This is given by the steady-state distribution, $u_{*}(x)$, 
which is the solution to the following ordinary differential equation (ODE)

$\frac{\tau}{2} \frac{\mathrm{d}^{2}}{\mathrm{~d} x^{2}}\left[D(x) u_{*}(x)\right]-\frac{\mathrm{d}}{\mathrm{d} x}\left[c(x) u_{*}(x)\right]+\frac{\tau}{2} \frac{\mathrm{d}}{\mathrm{d} x}\left[c(x) \frac{\mathrm{d} c}{\mathrm{~d} x} u_{*}(x)\right]=0,(13)$

obtained by setting the left-hand side of Eq. 9 to zero. Notice that Eq. 13 does not depend upon the rescaling constant $\epsilon$. The solution to Eq. 13 with boundary conditions derived from Eq. 12 is given as follows [this result is well-known; see, e.g., Moorcroft and Barnett (2008, Appendix A) for a derivation in the context of animal movement]

$u_{*}(x)=\frac{C_{H}}{D(x)} \exp \left[\frac{1}{\tau} \int_{0.5}^{x} \frac{2 c(s)-\tau \frac{\mathrm{d} c}{\mathrm{~d} s} c(s)}{D(s)} \mathrm{d} s\right]$,

where $C_{H}$ is a normalizing constant ensuring $u_{*}(x)$ integrates to 1 across the domain $[0,1]$.

For the movement kernel in our model (3), the integrals from Eqs. 10 and 11 can be calculated directly to be

$$
\begin{aligned}
& c(x)= \begin{cases}\frac{\sigma}{\tau \sqrt{2 \pi} g(x)}\left[(\beta-1) \exp \left(\frac{-(1-2 x)^{2}}{8 \sigma^{2}}\right)+\exp \left(\frac{-x^{2}}{2 \sigma^{2}}\right)\right. & \\
\left.-\beta \exp \left(\frac{-(1-x)^{2}}{2 \sigma^{2}}\right)\right] & \text { if } 0 \leq x \leq 1 / 2, \\
\frac{\sigma}{\tau \sqrt{2 \pi} g(x)}\left[(1-\alpha) \exp \left(\frac{-(1-2 x)^{2}}{8 \sigma^{2}}\right)+\alpha \exp \left(\frac{-x^{2}}{2 \sigma^{2}}\right)\right. & \\
\left.-\exp \left(\frac{-(1-x)^{2}}{2 \sigma^{2}}\right)\right] & \text { if } 1 / 2<x \leq 1,\end{cases} \\
& D(x)= \begin{cases}\frac{\sigma}{\tau^{2} \sqrt{2 \pi} g(x)}\left[-x \exp \left(\frac{-x^{2}}{2 \sigma^{2}}\right)\right. \\
-(1-\beta) \frac{1-2 x}{2} \exp \left(\frac{-(1-2 x)^{2}}{8 \sigma^{2}}\right) \\
+\sigma(1-\beta) \sqrt{\frac{\pi}{2}} \operatorname{erf}\left(\frac{1-2 x}{2 \sqrt{2} \sigma}\right)+\sigma \sqrt{\frac{\pi}{2}} \operatorname{erf}\left(\frac{x}{\sqrt{2} \sigma}\right) \\
-\beta(1-x) \exp \left(\frac{-(1-x)^{2}}{2 \sigma^{2}}\right) & \\
\left.+\sigma \beta \sqrt{\frac{\pi}{2}} \operatorname{erf}\left(\frac{1-x}{\sqrt{2} \sigma}\right)\right]-c(x)^{2} & \text { if } 0 \leq x \leq 1 / 2 \\
\frac{\sigma}{\tau^{2} \sqrt{2 \pi} g(x)}\left[-x \alpha \exp \left(\frac{-x^{2}}{2 \sigma^{2}}\right)\right. & \\
-(\alpha-1) \frac{1-2 x}{2} \exp \left(\frac{-(1-2 x)^{2}}{8 \sigma^{2}}\right) & \\
+\sigma(\alpha-1) \sqrt{\frac{\pi}{2}} \operatorname{erf}\left(\frac{1-2 x}{2 \sqrt{2} \sigma}\right)+\sigma \alpha \sqrt{\frac{\pi}{2}} \operatorname{erf}\left(\frac{x}{\sqrt{2} \sigma}\right) & \\
-(1-x) \exp \left(\frac{-(1-x)^{2}}{2 \sigma^{2}}\right) & \\
\left.+\sigma \sqrt{\frac{\pi}{2}} \operatorname{erf}\left(\frac{1-x}{\sqrt{2} \sigma}\right)\right]-c(x)^{2} & \text { if } 1 / 2<x \leq 1\end{cases}
\end{aligned}
$$

where $\operatorname{erf}(-)$ is the error function and

$$
\begin{aligned}
g(x) & =\frac{1}{\sigma \sqrt{2 \pi}} \int_{0}^{1} \exp \left[(z-x)^{2} / 2 \sigma^{2}\right] W[H(z), H(x)] \mathrm{d} z \\
& =\left\{\begin{array}{l}
\frac{1}{2}\left[(1-\beta) \operatorname{erf}\left(\frac{1-2 x}{2 \sqrt{2} \sigma}\right)+\operatorname{erf}\left(\frac{x}{\sqrt{2} \sigma}\right)+\beta \operatorname{erf}\left(\frac{1-x}{\sqrt{2} \sigma}\right)\right] \text { if } 0 \leq x \leq 1 / 2, \\
\frac{1}{2}\left[(1-\alpha) \operatorname{erf}\left(\frac{2 x-1}{2 \sqrt{2} \sigma}\right)+\alpha \operatorname{erf}\left(\frac{x}{\sqrt{2} \sigma}\right)+\operatorname{erf}\left(\frac{1-x}{\sqrt{2} \sigma}\right)\right] \text { if } 1 / 2<x \leq 1 .
\end{array}\right.
\end{aligned}
$$

Technical note In Fig. 2, we see that the diffusion coefficient $D(x)$ can be non-differentiable at the transition point 0.5 , while the drift velocity $c(x)$ can have a jump at 0.5 . Hence our limiting Eq. 13 has non-smooth coefficients and so solutions to Eq. 13 must be understood in the weak sense. In Appendix B, we outline how weak solutions are defined in our case and show that the explicit solution (14) is a weak solution, as long as $D(x)$ is continuous. If $D(x)$ is continuous, as in the examples in Fig. 2e-g, the solution at $x=0.5$ is simply $u_{*}(0.5)=C_{H} / D(0.5)$. However, in certain cases not studied here (e.g., if $\alpha, \beta>1$ but $\alpha \neq \beta), D(x)$ is discontinuous at $x=0.5$, so $u_{*}(x)$ is discontinuous at $x=0.5$, so Eq. 14 is no longer a weak solution in the sense described on Appendix B. These cases are thus not amenable to the exact analysis described here. 
Equations 14-17 give the predicted population abundance patterns that emerge from the movement kernel in Eq. 3. Though ostensibly formidable, Eqs. 15-17 are simply sums of well-known special functions, so can be calculated very quickly with a desktop computer.

In Fig. 3, we show examples of the steady-state solution (14). We examine qualitatively the effects of varying $\alpha, \beta$, and $\sigma$, including cases where $\alpha, \beta<1, \alpha, \beta>1$ and $\alpha<1<\beta$, comparing them to situations found in nature. Positive, negative, and transitional edge effects emerge, depending on the values chosen for $\alpha$ and $\beta$. Furthermore, the width of these edge effects, observed at the centre of each plot, increases as the distance an animal tends to move between movement decisions, given by $\sigma$, increases (Figs. 3a, d, 4).

\section{Comparison with other approaches}

In this section, we describe two slightly different approaches to constructing a PDE model from a movement kernel. The first starts with the integro-PDE of Eq. 7 and proceeds via a moment closure method. This is just a simple modification of the hyperbolic scaling in section "A partial differential equation approximation" so bears some attention. The second was introduced by Patlak (1953) and takes a first-principles approach (though the resulting algebraic manipulations are far from simple). Since its popularization by Turchin (1991), Patlak's work has been widely used to study animal movement, so is worth examining here. We compare the steady state solutions from these approaches with those of the hyperbolic scaling.

\section{The moment closure approach}

The moment closure approach begins with the integro-PDE of Eq. 7 but, unlike the hyperbolic scaling, requires no rescaling (see Eq. 8). However, it makes a different (and somewhat stronger) set of assumptions. In particular, Hillen and Painter (2013) showed that if

- the momentum of the object settles to its equilibrium distribution relatively quickly,

- the "pressure tensor" $u(t, x) D(x)$ and "energy flow" $u(t, x) M_{3}(x) / \tau^{3}$ are at equilibrium (where $M_{3}(x)$ is the third moment of the displacement distribution)

then the system given by Eq. 7 can be approximately described by the following equation

$\frac{\partial u}{\partial t}=\frac{\tau}{2} \frac{\partial^{2}}{\partial x^{2}}[D(x) u(x, t)]-\frac{\partial}{\partial x}[c(x) u(x, t)]$.
The steady-state ODE is therefore

$\frac{\tau}{2} \frac{\mathrm{d}^{2}}{\mathrm{~d} x^{2}}\left[D(x) u_{*}(x)\right]-\frac{\mathrm{d}}{\mathrm{d} x}\left[c(x) u_{*}(x)\right]=0$,

which, by assuming zero-flux boundary conditions at $x=$ 0,1 , has the following solution

$u_{*}(x)=\frac{C_{M}}{D(x)} \exp \left[\frac{2}{\tau} \int_{0.5}^{x} \frac{c(s)}{D(s)} \mathrm{d} s\right]$

where $C_{M}$ is a normalizing constant ensuring $u_{*}(x)$ integrates to 1 across the domain $[0,1]$. This solution only differs from the hyperbolic scaling solution (14) in that the term $\tau(\mathrm{d} c / \mathrm{d} s) c(s) / 2$ is no longer present in the integrand.

\section{Patlak's approach}

Patlak (1953) modeled animal movement making straightline movements (steps) interspersed with turns. The direction of a step may be correlated with the previous step, and there may be some external bias influencing the steplengths and turning angles. The strength and direction of this bias may vary through space. Let $\tau$ denote the average time between successive turns (as is the case in Eq. 1, for example). Then (Patlak 1953, equation 19ff.) showed that the following PDE holds approximately

$$
\frac{\partial u}{\partial t}(x, t)=\frac{1}{d} \nabla_{x}^{2}\left[\frac{M_{2}(x)}{2 \tau} u(x, t)\right]-\nabla_{x} \cdot\left[\frac{M_{1}(x)}{\tau} u(x, t)\right],
$$

where $d$ is the number of dimensions in which the animal is moving, $M_{2}(x)$ is the second moment of the step-length distribution and $M_{1}(x)=\left(M_{1}\left(x_{1}\right), \ldots, M_{1}\left(x_{d}\right)\right)$ is a vector with $i$ th entry equal to the first moment of the distance moved between turns in the $i$ th direction. The derivation of Eq. 21 relies on the assumption that the bias in the movement is not too strong [for a precise explanation of what "too strong" means in this context, see equations (11) and (16) in Patlak (1953) and the surrounding text]. A similar result to Eq. 21 is found earlier, in Chandresekhar (1943, equation 126). In our model $d=1$, so we make this assumption for the rest of the section.

A key contribution of Patlak (1953) was to analyze the term $M_{1}(x)$ for objects moving with certain types of bias and persistence. This analysis gave rise to general formulae which have since been popularized in the ecological literature by Turchin (1991, e.g., equation 1) and used widely in movement ecology (Turchin 1998). However, 
Fig. 2 Examples of the drift and diffusion terms. Plots of the drift term (a-d) given in Eq. 15 and the diffusion term $(\mathbf{e}-\mathbf{g})$ in Eq. 16, for various values of $\alpha, \beta, \sigma$
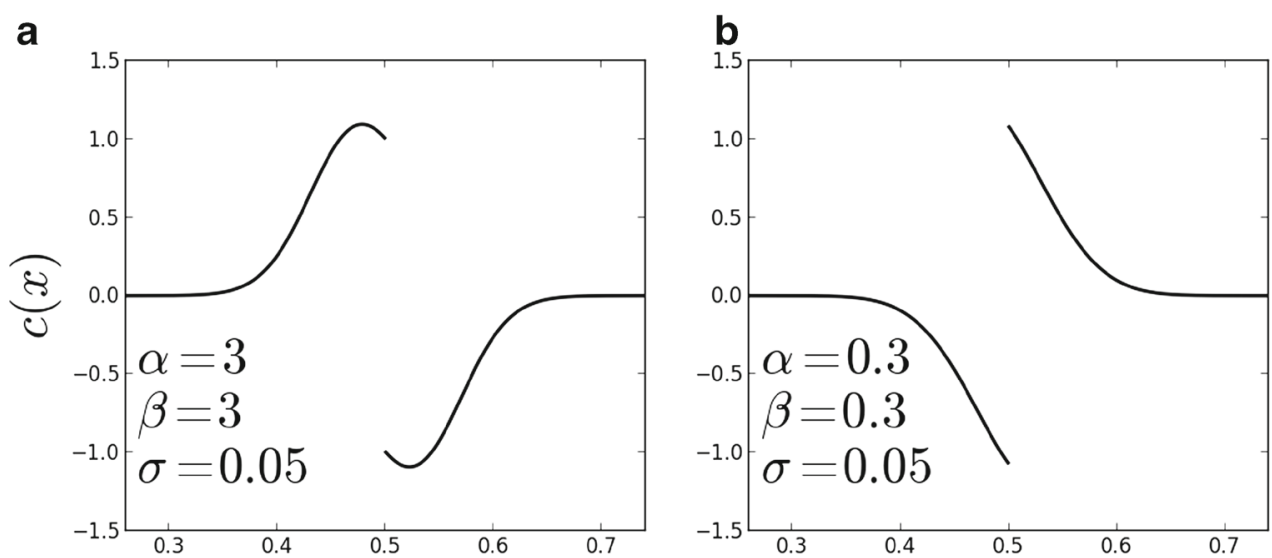

C

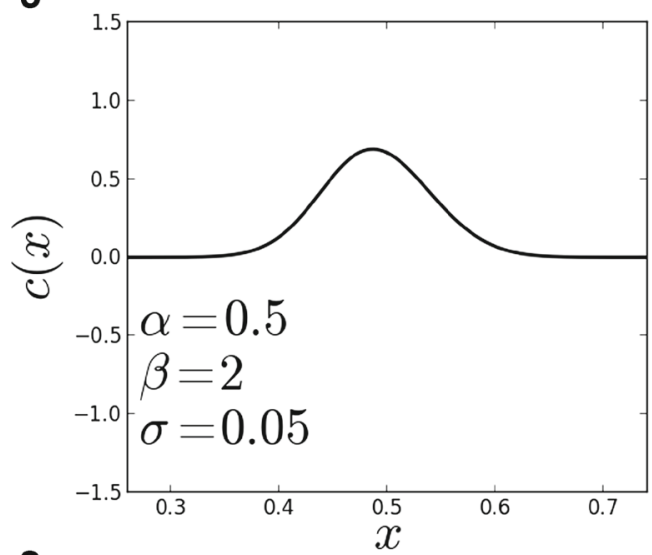

e

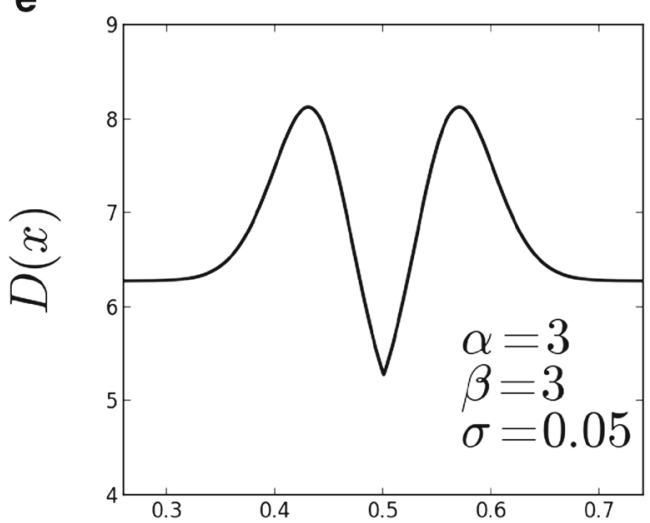

g

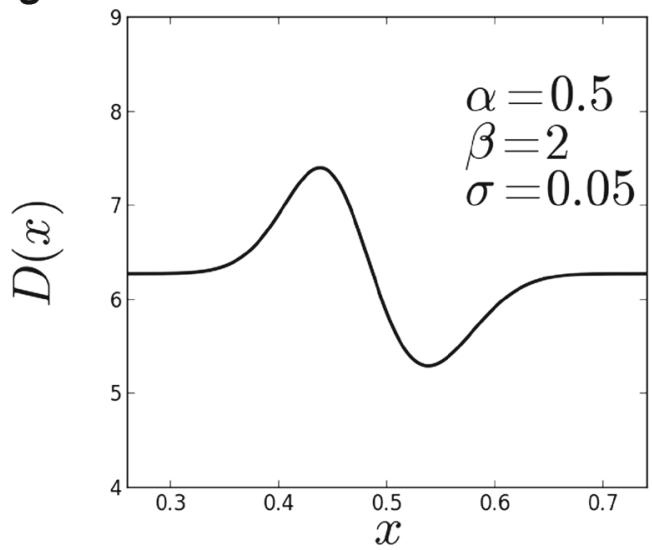

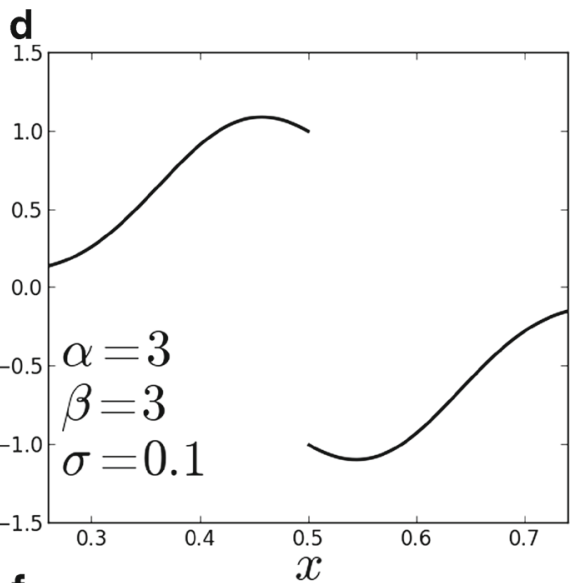

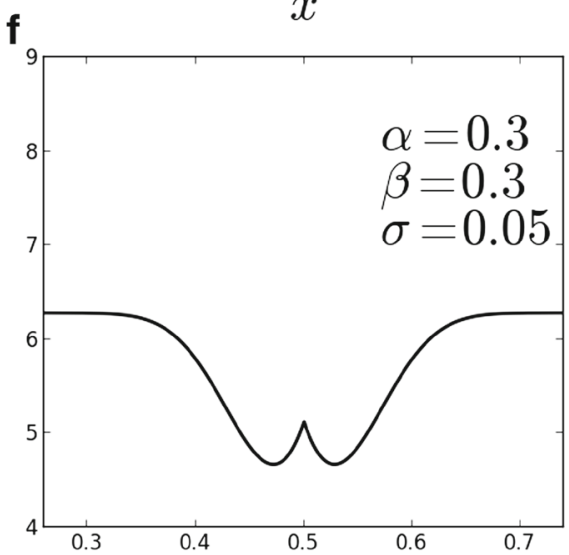

h

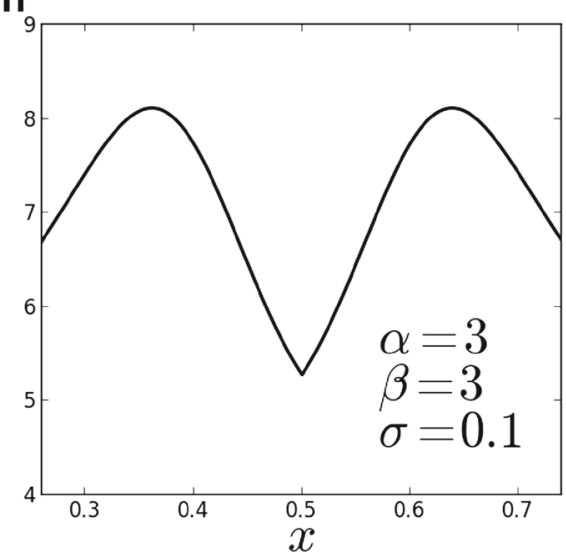



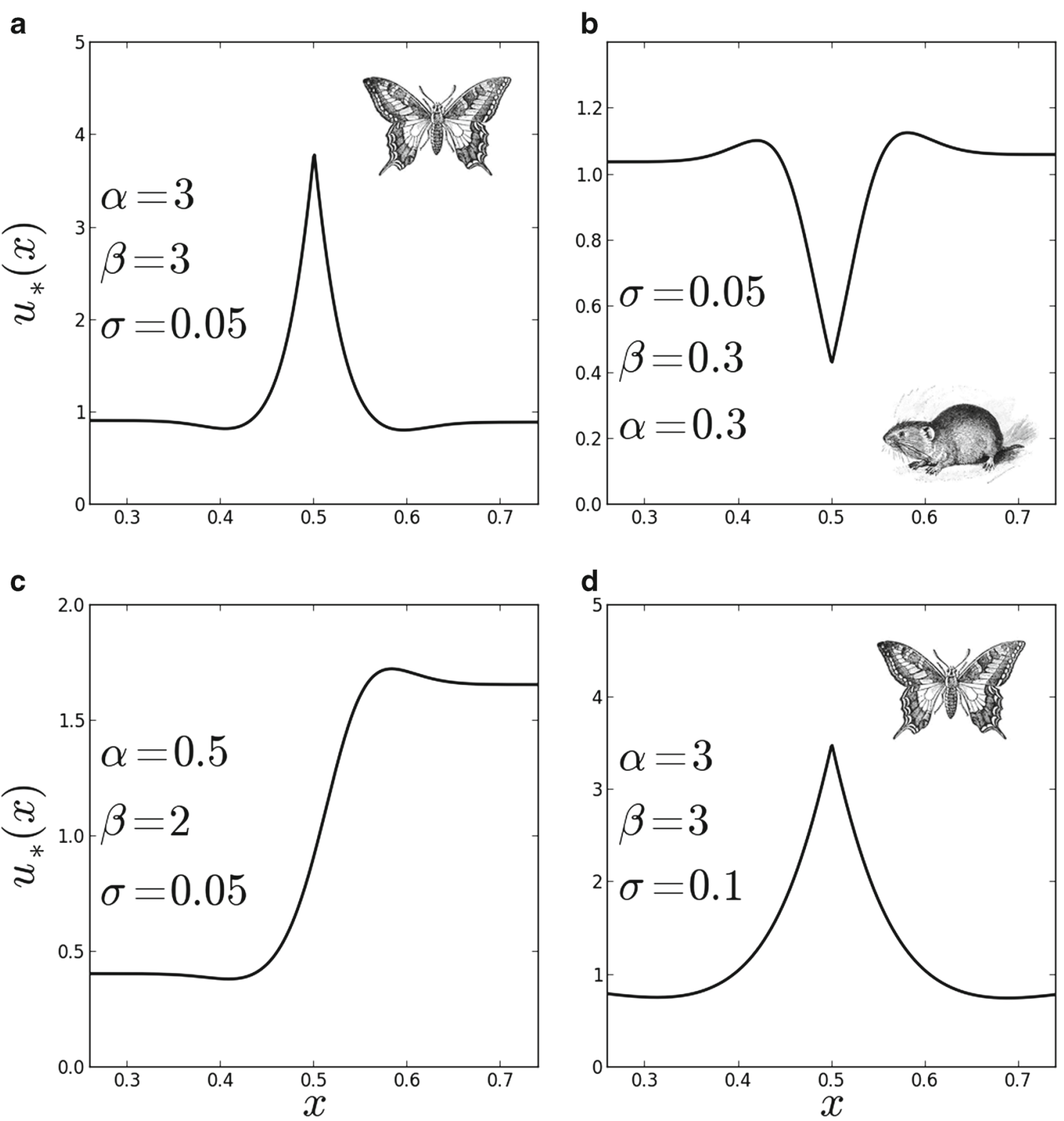

Fig. 3 Emergent edge effects. The steady-state position distributions from Eq. 14 for various values of $\sigma$ (measuring the distance an animal tends to move in a timestep of length $\tau$ ), $\alpha$ (the weighting for moving from the right-half to the left), and $\beta$ (the weighting for moving from the left-half to the right). Notice that if both $\alpha$ and $\beta$ are greater then 1 then a positive edge effect emerges where animals are drawn towards the edge. This has been observed in a variety of population, for

example the butterfly populations studied by Batáry et al. (2009). Conversely, if $\alpha, \beta<1$ then a negative edge effect emerges. An example of this is if the edge is delineated by a road separating habitats inhabited by small mammals (Goosem 2001). The butterfly and vole images are from http://etc.usf.edu/clipart/, licensed under the Free Classroom License http://etc.usf.edu/clipart/info/license

instead of using these derived formulae, we can calculate Similarly, $M_{2}(x)$ is calculated as $M_{1}(x)$ directly from the movement kernel of Eq. 3, as follows

$M_{1}(x)=\int_{0}^{1}(z-x) k_{\tau}(z \mid x) \mathrm{d} z$.

$M_{2}(x)=\int_{0}^{1}(z-x)^{2} k_{\tau}(z \mid x) \mathrm{d} z$ 


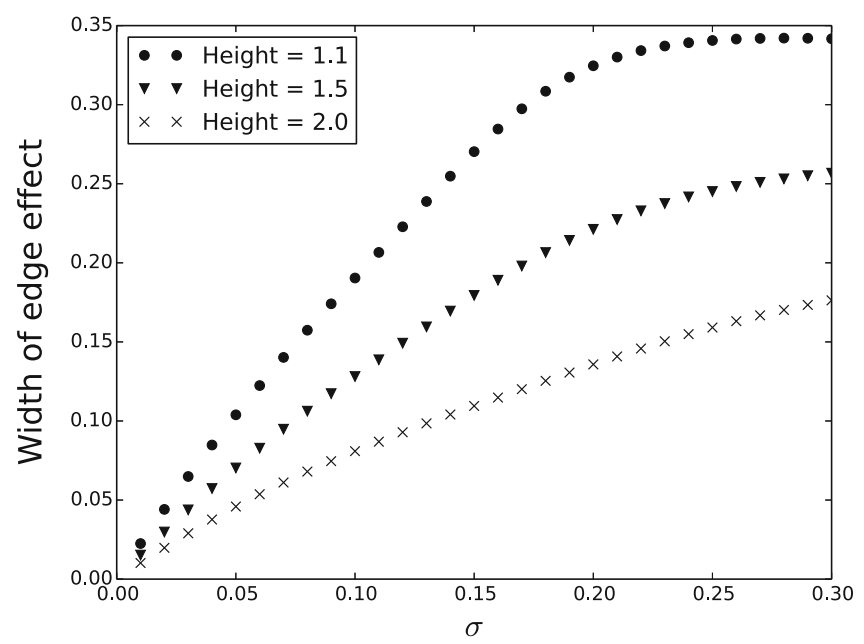

Fig. 4 Edge effect widens with increasing $\sigma$. As we increase the distance that the animal tends to move between successive movement bouts, represented by $\sigma$, so the width of the resulting edge effect in the population abundance patterns increases. The plot shows this width for $\alpha=\beta=3$ at different heights $h$ of the utilisation distribution (see

The steady-state solution to Eq. 21 is obtained by setting the left-hand side of Eq. 21 to zero. In 1D, this gives the following ODE

$\frac{\mathrm{d}^{2}}{\mathrm{~d} x^{2}}\left[\frac{M_{2}(x)}{2 \tau} u_{*}(x)\right]-\frac{\mathrm{d}}{\mathrm{d} x}\left[\frac{M_{1}(x)}{\tau} u_{*}(x)\right]=0$,

where $u_{*}(x)$ is the steady-state of $u(x, t)$. By applying zero-flux boundary conditions at $x=0,1, u_{*}(x)$ has the following expression

$u_{*}(x)=\frac{C_{P}}{M_{2}(x)} \exp \left[\int_{0.5}^{x} \frac{2 M_{1}(s)}{M_{2}(s)} \mathrm{d} s\right]$,

where $C_{P}$ is a normalizing constant ensuring $u_{*}(x)$ integrates to 1 across the domain [0, 1].

The integrals from Eqs. 22 and 23 can be related to the expressions in Eqs. 15 and 16 using the following identities

$M_{1}(x)=\tau c(x)$,

$M_{2}(x)=\tau^{2} D(x)+M_{1}(x)^{2}$.

Hence an exact analytic expression for the steady-state solution in Eq. 25 can be written.

Comparing the hyperbolic rescaling solution (14, 26, and 27) to the solution using Patlak's formalism (25), we see the following differences:

- The second moment of the velocity in Patlak's formalism is replaced by the variance in the hyperbolic rescaling.
Fig. 3a, d). This width is calculated by taking the two points where $u_{*}(x)=h$ and finding the distance between them. Notice that for low $\sigma$ (below about 0.05 ), this effect is roughly linear. For larger $\sigma$, the non-linearity likely comes about from the effect of the landscape edges at positions 0 and 1

- The first moment of the velocity in Patlak's formalism is supplemented by an additional term, $\frac{\tau}{2} \frac{\mathrm{d} c}{\mathrm{~d} x} c(x)$, in the hyperbolic rescaling.

\section{Three-way comparison}

In Fig. 5, we compare Eqs. 14, 20, and 25 which describe steady-state results using the three different formalisms, for various different values of $\alpha$ and $\beta$ from our model described in Fig. 1. Qualitatively, the results are very similar. For each of the models, a tendency to cross the edge in both directions leads to higher population abundance near the edge. Conversely, a tendency not to cross the edge in either direction leads to lower population abundance near the edge. Furthermore, the width of the peak or trough in population abundance is positively correlated to the distance over which the animal tends to move each movement bout (represented by $\sigma$ ). Thus, we can connect analytically certain behavioral features of the animal (e.g., the extent over which it travels between movement decisions) to population-level patterns near edges.

Despite these similarities between models, there are also some slight quantitative differences. Particularly, the TE approaches give more pronounced edge effects in the case where the effect is positive. These differences can be understood by analyzing the steady-state ODEs for the three models. Define $\mathcal{M}$ to be the left-hand side of the moment closure steady-state ODE in Eq. 19 . Define $\mathcal{P}$ to be the analogous expression for Patlak's model [the left-hand side of 
Fig. 5 Comparison of three PDE approximations. The steady-state position distributions from Eqs. 14 (the hyperbolic scaling model; grey dashed curves), 20 (the moment closure model; grey solid curves), and 25 (Patlak's model; black curves) for the various values of $\sigma, \alpha$, and $\beta$ plotted in Fig. 3 a

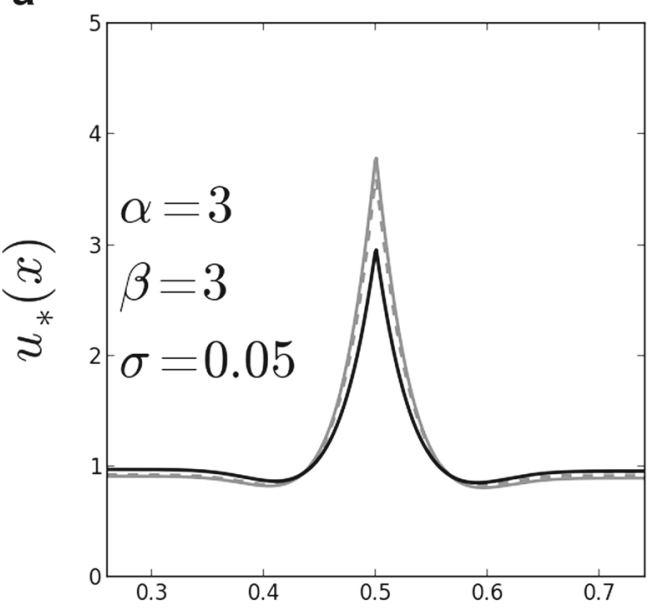

C

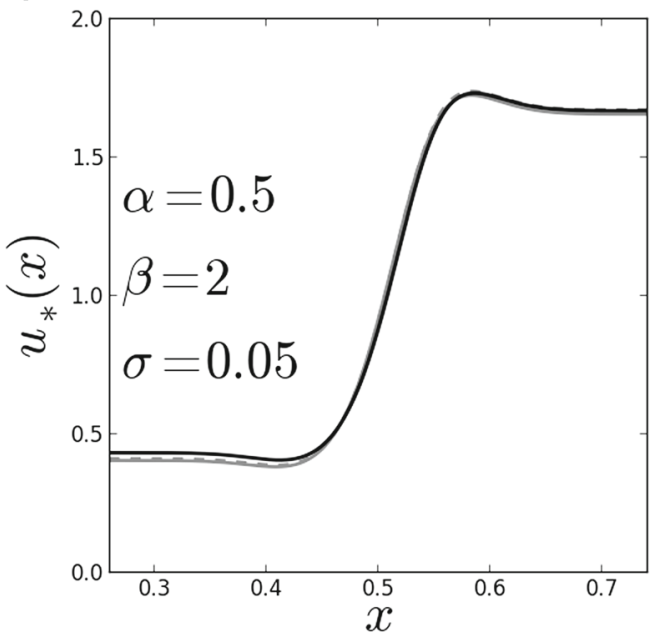

b

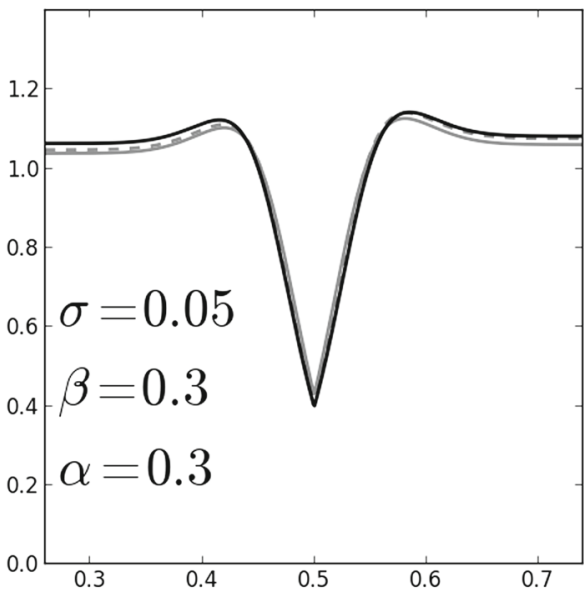

d

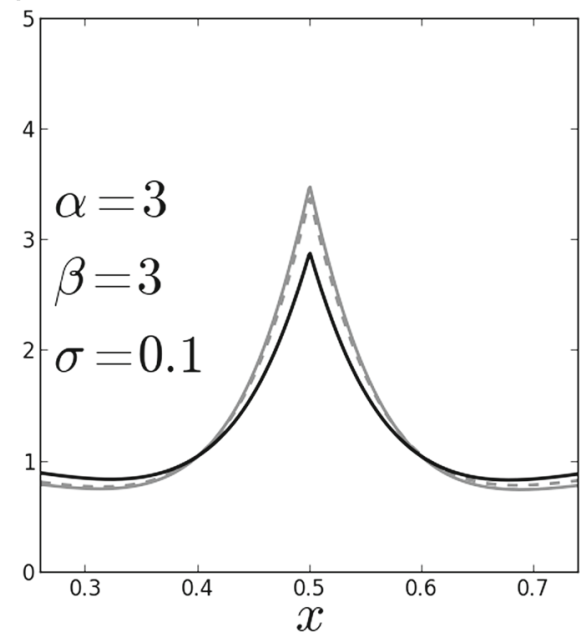

Eq. 24]. Define $\mathcal{H}$ to be the analogous expression for the hyperbolic scaling [the left-hand side of Eq. 13]. Then

$\mathcal{P}=\mathcal{M}+\frac{\tau}{2} \frac{\mathrm{d}^{2}}{\mathrm{~d} x^{2}}\left[c^{2}(x) u_{*}(x)\right]$,

$\mathcal{H}=\mathcal{M}+\frac{\tau}{2} \frac{\mathrm{d}}{\mathrm{d} x}\left[c(x) \frac{\mathrm{d} c}{\mathrm{~d} x} u_{*}(x)\right]$.

Equations 28 and 29 show that Patlak's model includes an additional (positive) diffusion term not present in the other two. This has the effect of decreasing the gradient at any point in the steady-state solution. This is precisely what we observe in Fig. 5.

Equation 29 shows that the difference between the moment closure expression and the hyperbolic scaling occurs in the advection term. Namely, the advection term in $\mathcal{H}$ is less than that in $\mathcal{M}$ by an amount equal to $\left[c(x) c^{\prime}(x) u_{*}(x)\right]^{\prime}$ (here, the apostrophe denotes differentiation with respect to $x$ ). Decreasing the advection term causes gradients to decrease, which is observed in the difference between the dashed and solid grey curves in Fig. 5. In our examples, the term $\left[c(x) c^{\prime}(x) u_{*}(x)\right]^{\prime}$ is small compared to $c(x)$, so its effect on the steady-state curves is minimal.

\section{Discussion and conclusions}

We have shown how population abundance patterns at edges emerge from edge-crossing decisions encoded in a movement kernel. The distance over which an animal makes habitat selection decisions turns out to be an important factor governing the resulting patterns (Fig. 4). Animals making decisions locally will exhibit minimal edge effect 
compared to those who make decisions based on greater movement distances. Indeed, if the infinitesimal limit $\sigma \rightarrow$ 0 were taken in Eqs. 15-17, then this edge effect would vanish completely. As well as enabling derivation of predicted population patterns from movement data, one could potentially use our results to solve the reverse problem: fitting the steady-state patterns from our models to infer the underlying parameters $(\alpha, \beta, \sigma)$ that govern the movement.

We have detailed three different methods to analyze the movement kernel. All of them give qualitatively similar results, but there are some small numerical differences owing to the different assumptions being made. In particular, the positive edge effect is less pronounced when using the approach of Patlak (1953), since that approach assumes that any bias in movement is minimal. In contrast, the positive edge effect arises from having a large crossing bias near the edge. However, for many ecological purposes, the numerical differences are likely too small to be of practical importance.

Although we have focussed on a very simple model landscape, simple enough to gain analytic insight, our model setup can readily be extended into more complex scenarios and higher dimensions. The review by Hillen and Painter (2013) gives mathematical details for deriving the hyperbolic limit PDE in a general case, and there are numerous examples of more complicated movement kernels that can take into account the various landscape features encountered by animals (e.g., Rhodes et al. 2005; Forester et al. 2009; Potts et al. 2014a), or the fact that animals may move at different speeds in different habitats (i.e., $\sigma$ becomes $\sigma(x)$ ). However, in such scenarios, it is likely that the resulting PDE could only be solved numerically and it would be difficult to disentangle the effects of different landscape features on the spatial patterns. Our study, of a deliberately simplified model, may help interpret aspects of spatial patterns that emerge in more complicated models. Indeed, edge effects were observed in the model of Potts et al. (2014a), and the present study helps interpret this in terms of the relative strengths of selection to move between habitat types.

There are, of course, certain limitations in a simple modeling approach that need to be addressed. Though the spatial scales inherent in movement decisions appear to play an important role in emergent edge effects, it is important to note that only one type of decision has been modeled here: that related to choice of habitat when close to an edge. In reality, animals may be making decisions on multiple scales and these all have an effect on their movement and hence the population patterns that emerge. Our focus purely on habitat selection decisions means that one could only expect the results of this paper to apply in scenarios where such selections are the primary drivers of movement. For example, a data set that includes migratory movement in addition to movement between different habitats may exhibit rather different patterns to those observed here. As another example, daily behavioral features such as movement to and from a sleeping place would affect the steady-state patterns (Moorcroft and Lewis 2006). Such examples would necessitate further analysis, modifying the techniques described in this study to account for such other behavioral features.

As well as answering the fundamental question of how population-level patterns may emerge from behavioral process, our analysis makes it possible for ecologists to test whether observed edge-crossing behavior is sufficient for explaining the population-level phenomena, or whether there is some other aspect of behavior causing the population-level effect. For certain species, it has been conjectured that population abundance may be higher on edges due to the unique habitat they provide (Didham and Lawton 1999; Batáry et al. 2009; Macreadie et al. 2010; Andren and Angelstam 1988). Alternatively, populationlevel patterns could arise from a combination of these factors, together with edge-crossing tendencies. Disentangling the various behavioral mechanisms that may give rise to edge patterns is a key goal for behavioral- and landscapeecology. The mathematical analysis provided here may help with this endeavor, by deriving expected population patterns that may emerge purely from edge-crossing decisions, then comparing them to the observed population patterns. Any disparity between predicted and observed would suggest that the edge-crossing decisions are not the sole driver of the population patterns, and hence some other effect is in play.

Much previous theoretical work on edge effects has been statistical in nature, asking how we determine the nature of edge effects rather than why edge effects emerge (Fernández et al. 2002; Zheng and Chen 2000). Though mechanistic approaches have been proposed, the models tend to be constructed from observed behavioral tendencies, then fit to data on the population-level, rather than rigorously derived from underlying movement decisions. For example, Tyson et al. (2007) looked the positive edge effects in moth populations elicited by attraction to an orchard (the edge) between two fields. They modeled moths via an advection-diffusion equation, with a drift tendency towards the orchard. Similarly, Fortin et al. (2013) used an advection-diffusion approach to examine how populations can cluster near anthropogenically disturbed habitats. However, in both studies, the advection-diffusion models are not rigorously derived from the underlying behavioral mechanisms, despite the fact that small changes in such mechanisms can lead to very different macroscopic patterns 
(e.g., Belmonte-Beitia et al. 2013). Our study explicitly makes such derivations, so could be used to parameterize models such as those of Tyson et al. (2007) or Fortin et al. (2013) from fine-scale details of the animals' movements.

The effect of edges on population spread has also received some rigorous theoretical treatment in recent years. Ovaskainen and Cornell (2003) derived advection diffusion equations in situations where the edge crossing probability causes a bias in movement. A more general treatment of diffusion through heterogeneous landscapes followed a few years later (Ovaskainen 2008). Since then, these tools have been successfully applied and extended in a variety of ways (Reeve et al. 2008; Zheng et al. 2009; Reeve and Cronin 2010; Xiao et al. 2013). On a similar vein, but using a slightly different approach, Hillen et al. (2013) examined situations where the density of a diffusing population can blow-up on the edges of a corridor through a landscape. However, all these studies rely on taking infinitesimally small-scale limits of the underlying random walk. Though this is a reasonable strategy when edge effects are narrow compared to the width of habitat patches, our study shows that these techniques may become inaccurate in landscapes where the distance travelled in each movement bout is not significantly smaller than the size of habitat patches.

The results given here could also provide insights into the general mechanistic framework of Ries et al. (2004) for understanding edge effects. That study examined four key factors that underlie edge effects, characterized as (i) ecological flows, (ii) access, (iii) resource mapping, and (iv) species interactions. However, they do not make explicit the movement decisions that either affect or are affected by these factors. The techniques given in this study could help integrate these fine-scale decisions into the mechanistic framework of Ries et al. (2004) and thus enhance their predictive power.

In summary, our work provides for the first time (as far as we are aware) an analytical link between the behavioral decisions of individual animals near edges and the sort of emergent population abundance patterns often observed in nature. The techniques provided here should aid in understanding why these observed population patterns emerge. As the ability of behavioral models to accurately represent reality increases (Potts et al. 2014b), such techniques will ultimately help predict population demographics in potential future environments.

Acknowledgments This study was partly funded by NSERC Discovery (TH, MAL) and Accelerator (MAL) grants. MAL also gratefully acknowledges a Canada Research Chair and a Killam Research Fellowship. We are grateful to Greg Breed and members of the Lewis Lab for helpful discussions, as well as anonymous reviewers whose comments helped improve the manuscript.
Open Access This article is distributed under the terms of the Creative Commons Attribution 4.0 International License (http:// creativecommons.org/licenses/by/4.0/), which permits unrestricted use, distribution, and reproduction in any medium, provided you give appropriate credit to the original author(s) and the source, provide a link to the Creative Commons license, and indicate if changes were made.

\section{Appendix A}

Hillen and Painter (2013) showed that the following PDE is a good approximation of Eq. 7 when the rescaling from Eq. 8 is made

$$
\begin{aligned}
\frac{\partial u}{\partial \vartheta}(\xi, \vartheta)= & \frac{\epsilon}{\mu} \frac{\partial^{2}}{\partial \xi^{2}}[\tilde{D}(\xi) u(\xi, \vartheta)]-\frac{\partial}{\partial \xi}[\tilde{c}(\xi) u(\xi, \vartheta)] \\
& +\frac{\epsilon}{\mu} \frac{\partial}{\partial \xi}\left[\tilde{c}(\xi) \frac{\partial \tilde{c}(\xi)}{\partial \xi} u(\xi, \vartheta)\right],
\end{aligned}
$$

where $\tilde{c}(\xi)$ denotes the expectation of $\tilde{q}(\xi, v)[\tilde{q}(\xi, v)$ is the turning kernel in units of $\xi$, see Eq. 6]

$\tilde{c}(\xi)=\int_{V(\xi)} v \tilde{q}(\xi, v) \mathrm{d} v$

and $\tilde{D}(\xi)$ is the variance of $\tilde{q}(\xi, v)$

$\tilde{D}(\xi)=\int_{V(\xi)}(v-\tilde{c})^{2} \tilde{q}(\xi, v) \mathrm{d} v$.

The set $V(\xi)$ denotes the set of all possible velocities. To write down $V(\xi)$ explicitly, it helps to translate Eq. 4 into $(\xi, \vartheta)$-coordinates to give

$\tilde{q}(\xi, v)=\epsilon \tau \tilde{k}_{\epsilon \tau}(\xi+\tau \epsilon v \mid \xi)$,

with

$\tilde{k}_{\epsilon \tau}(\zeta \mid \xi)=\frac{k_{\tau}(z \mid x)}{\epsilon}, \quad z=\frac{\zeta}{\epsilon}, \quad x=\frac{\xi}{\epsilon}$.

In our model, the velocities are limited so that the animal cannot move outside the interval $[0, \epsilon]$ in a single timestep of length $\epsilon \tau$, in the rescaled units. Since $\xi \in[0, \epsilon]$, we thus have $V(\xi)=[-\xi /(\epsilon \tau),(\epsilon-\xi) /(\epsilon \tau)]$. The choice of $\tilde{q}(\xi, v)$ in Eq. 33 then leads to

$$
\begin{aligned}
\tilde{c}(\xi) & =\int_{V} v \tilde{q}(\xi, v) \mathrm{d} v \\
& =\int_{0}^{\epsilon} \frac{\zeta-\xi}{\epsilon \tau}\left[\epsilon \tau \tilde{k}_{\epsilon \tau}(\zeta \mid \xi)\right] \frac{\mathrm{d} \zeta}{\epsilon \tau} \\
& =\frac{1}{\epsilon \tau} \int_{0}^{\epsilon}(\zeta-\xi) \tilde{k}_{\epsilon \tau}(\zeta \mid \xi) \mathrm{d} \zeta \\
& =\frac{1}{\epsilon \tau} \int_{0}^{1}(\epsilon z-\epsilon x) \frac{k_{\tau}(z \mid x)}{\epsilon} \epsilon \mathrm{d} z \\
& =c(x),
\end{aligned}
$$


where $c(x)$ is the expectation of the velocity in the original $(x, t)$ co-ordinates. Similarly, we obtain for $\tilde{D}(\xi)$

$$
\begin{aligned}
\tilde{D}(\xi)= & \int_{V}(v-\tilde{c})^{2} \tilde{q}(\xi, v) \mathrm{d} v \\
= & \int_{0}^{\epsilon} \frac{(\zeta-\xi-\tau \epsilon \tilde{c})^{2}}{\epsilon^{2} \tau^{2}}\left[\epsilon \tau \tilde{k}_{\epsilon \tau}(\zeta \mid \xi)\right] \frac{\mathrm{d} \zeta}{\epsilon \tau} \\
= & \frac{1}{\epsilon^{2} \tau^{2}} \int_{0}^{\epsilon}(\zeta-\xi)^{2} \tilde{k}_{\epsilon \tau}(\zeta \mid \xi) \mathrm{d} \zeta \\
& -\frac{2}{\epsilon \tau} \int_{0}^{\epsilon}(\zeta-\xi) \tilde{c}(\xi) \tilde{k}_{\epsilon \tau}(\zeta \mid \xi) \mathrm{d} \zeta+\tilde{c}(\xi)^{2} \\
= & \frac{1}{\epsilon^{2} \tau^{2}} \int_{0}^{\epsilon}(\zeta-\xi)^{2} \tilde{k}_{\epsilon \tau}(\zeta \mid \xi) \mathrm{d} \zeta-\tilde{c}(\xi)^{2} \\
= & \frac{1}{\epsilon^{2} \tau^{2}} \int_{0}^{1}(\epsilon z-\epsilon x)^{2} \frac{k_{\tau}(z \mid x)}{\epsilon} \epsilon \mathrm{d} z-c(x)^{2} \\
= & D(x)
\end{aligned}
$$

where $D(x)$ is the variance of the velocity in the original co-ordinates. Notice that $c(x)$ and $D(x)$ vary with space, but not time. Using the quantities $c(x)$ and $D(x)$, we can transform Eq. 30 back into the original co-ordinates. The result is given in Eq. 9.

\section{Appendix B}

We have seen that the coefficients $c(x)$ and $D(x)$ can be non-differentiable at 0.5 , and $c(x)$ can even have a jump. Assuming $D(x)$ is continuous, as in the examples of Fig. 3, the steady-state solutions are also continuous at 0.5 , but in some cases they can be non-differentiable. To deal with this property, we use the following definition of a weak solution.

Definition 1 Let $W^{1,2}([0,1])$ denote the Sobolev space of functions on $[0,1]$ that live in $L^{2}$-space and whose weak derivatives are also in $L^{2}$. We call $u_{*} \in W^{1,2}([0,1])$ a weak solution of Eq. 13, with zero-flux boundary conditions (12), if for all test functions

$\phi \in\left\{C^{2}([0,1]) ; \phi(0.5)=0, \phi^{\prime}(0)=\phi^{\prime}(0.5)=\phi^{\prime}(1)=0\right\}$

we have

$$
\begin{gathered}
-\frac{\tau}{2} \int_{0}^{1} \frac{\partial}{\partial x}\left(D u_{*}\right) \frac{\partial \phi}{\partial x} d x+\int_{0}^{1} c u_{*} \frac{\partial \phi}{\partial x} d x \\
+\frac{\tau}{4} \int_{0}^{1} c^{2} \frac{\partial}{\partial x}\left(u_{*} \frac{\partial \phi}{\partial x}\right) d x=0 .
\end{gathered}
$$

Lemma 1 The explicit solution given in Eq. 14 is a weak solution of Eq. 13 as long as $D(x)$ is continuous.
Proof The lemma can be shown by direct substitution of Eq. 14 into the definition of a weak solution.

It should be noted that the choice of test function $\phi$ with $\phi(0.5)=\phi^{\prime}(0.5)=0$ allows for an exceptional point at 0.5 and it would be expected that the solution is not unique at that point. However, by stipulating that $u_{*} \in W^{1,2}$, we guarantee that $u_{*}$ is continuous at 0.5 . Since we have the explicit solution (14), we know that such a solution exists, as long as $D(x)$ is continuous.

For the cases represented in Fig. 3, we can go further and show that the only possible solutions are continuous, regardless of the constraints put on $u_{*}$. We do this in two separate cases in the following.

Case $1 \alpha=1 / \beta$. In this case, $c(x)$ and $D(x)$ can be defined as the following smooth $\left(C^{\infty}\right)$ functions on $[0,1]$

$$
\begin{aligned}
c(x)= & \frac{\sigma \sqrt{2}}{\tau \sqrt{\pi} g(x)}\left[(\beta-1) \exp \left(\frac{-(1-2 x)^{2}}{8 \sigma^{2}}\right)\right. \\
& \left.+\exp \left(\frac{-x^{2}}{2 \sigma^{2}}\right)-\beta \exp \left(\frac{-(1-x)^{2}}{2 \sigma^{2}}\right)\right] \\
& \times\left[(1-\beta) \operatorname{erf}\left(\frac{1-2 x}{2 \sqrt{2} \sigma}\right)\right. \\
& \left.+\operatorname{erf}\left(\frac{x}{\sqrt{2} \sigma}\right)+\beta \operatorname{erf}\left(\frac{1-x}{\sqrt{2} \sigma}\right)\right]^{-1},
\end{aligned}
$$

$$
\begin{aligned}
D(x)= & \frac{\sigma \sqrt{2}}{\tau^{2} \sqrt{\pi} g(x)}\left[-x \exp \left(\frac{-x^{2}}{2 \sigma^{2}}\right)\right. \\
& -(1-\beta) \frac{1-2 x}{2} \exp \left(\frac{-(1-2 x)^{2}}{8 \sigma^{2}}\right) \\
& +\sigma(1-\beta) \sqrt{\frac{\pi}{2}} \operatorname{erf}\left(\frac{1-2 x}{2 \sqrt{2} \sigma}\right)+\sigma \sqrt{\frac{\pi}{2}} \operatorname{erf}\left(\frac{x}{\sqrt{2} \sigma}\right) \\
& -\beta(1-x) \exp \left(\frac{-(1-x)^{2}}{2 \sigma^{2}}\right) \\
& \left.+\sigma \beta \sqrt{\frac{\pi}{2}} \operatorname{erf}\left(\frac{1-x}{\sqrt{2} \sigma}\right)\right] \times\left[(1-\beta) \operatorname{erf}\left(\frac{1-2 x}{2 \sqrt{2} \sigma}\right)\right. \\
& \left.+\operatorname{erf}\left(\frac{x}{\sqrt{2} \sigma}\right)+\beta \operatorname{erf}\left(\frac{1-x}{\sqrt{2} \sigma}\right)\right]^{-1}-c(x)^{2} . \quad(38)
\end{aligned}
$$

Therefore, Eq. 9 can be defined on the whole interval [0, 1], so the steady-state solution from Eq. 14 is a classical solution valid on $[0,1]$. This is plotted for $\alpha=0.5, \beta=2$ in Fig. $3 c$ of the main text. 
Case $2 \alpha=\beta$. Here, we use a symmetry argument. Specifically, we observe that in this case the movement kernel $k_{\tau}(z \mid x)$ is invariant under change of co-ordinates $x \leftrightarrow 1-x$, $z \leftrightarrow 1-z$. It follows that $u_{*}(x)=u_{*}(1-x)$, i.e., $u_{*}(1 / 2+\delta)=u_{*}(1 / 2-\delta)$ for all $\delta \in(0,1 / 2]$. The only way this can be true is if the solution in Eq. 14 is continuous at $x=0.5$. The solution is plotted in Fig. $2 \mathrm{a}, \mathrm{b}, \mathrm{d}$ of the main text, for various parameter values where $\alpha=\beta$.

\section{References}

Andren H, Angelstam P (1988) Elevated predation rates as an edge effect in habitat islands: experimental evidence. Ecology 69(2):544-547

Barnett A, Moorcroft P (2008) Analytic steady-state space use patterns and rapid computations in mechanistic home range analysis. J Math Biol 57(1):139-159

Batáry P, Kõrösi A, Örvössy N, Kövér S, Peregovits L (2009) Species-specific distribution of two sympatric maculinea butterflies across different meadow edges. J Insect Conserv 13(2):223230

Belmonte-Beitia J, Woolley T, Scott J, Maini P, Gaffney E (2013) Modelling biological invasions: individual to population scales at interfaces. J Theor Biol 334(0): 1-12

Burkey TV (1989) Extinction in nature reserves: the effect of fragmentation and the importance of migration between reserve fragments. Oikos 55(1):75-81

Chandrasekhar S (1943) Stochastic problems in physics and astronomy. Rev Mod Phys 15:1-89

Didham RK, Lawton JH (1999) Edge structure determines the magnitude of changes in microclimate and vegetation structure in tropical forest fragments. Biotropica 31(1):17-30

Fernández C, Acosta FJ, Abellá G, López F, Díaz M (2002) Complex edge effect fields as additive processes in patches of ecological systems. Ecol Model 149(3):273-283

Forester J, Im H, Rathouz P (2009) Accounting for animal movement in estimation of resource selection functions: sampling and data analysis. Ecology 90:3554-3565

Fortin D, Beyer H, Boyce M, Smith D, Duchesne T, Mao J (2005) Wolves influence elk movements: behavior shapes a trophic cascade in yellowstone national park. Ecology 86:1320-1330

Fortin D, Buono PL, Fortin A, Courbin N, Gingras CT, Moorcroft PR, Courtois R, Dussault C (2013) Movement responses of caribou to human-induced habitat edges lead to their aggregation near anthropogenic features. Am Nat 181(6):827-836

Goosem M (2001) Effects of tropical rainforest roads on small mammals: inhibition of crossing movements. Wildl Res 28:351364

Hillen T (2006) M5 mesoscopic and macroscopic models for mesenchymal motion. J Math Biol 53(4):585-616

Hillen T, Painter K (2013) Transport and anisotropic diffusion models for movement in oriented habitats. In: Lewis MA, Maini PK, Petrovskii SV (eds) Dispersal, Individual Movement and Spatial Ecology, Lecture Notes in Mathematics, Springer Berlin Heidelberg, pp 177-222

Hillen T, Painter KJ, Winkler M (2013) Anisotropic diffusion in oriented environments can lead to singularity formation. Eur J Appl Math 24:371-413

Laurance SGW, Stouffer PC, Laurance WF (2004) Effects of road clearings on movement patterns of understory rainforest birds in Central Amazonia. Conserv Biol 18(4):1099-1109
Lidicker J, William Z (1999) Responses of mammals to habitat edges: an overview. Landsc Ecol 14(4):333-343

Macreadie PI, Hindell JS, Keough MJ, Jenkins GP, Connolly RM (2010) Resource distribution influences positive edge effects in a seagrass fish. Ecology 91(7):2013-2021

Manly B, McDonald L, Thomas D, McDonald T, Erikson W (2002) Resource selection by animals: statistical design and analysis for field studies. Elsevier Academic Press, Chapman and Hall, New York

McNeill SE, Fairweather PG (1993) Single large or several small marine reserves? An experimental approach with seagrass fauna. $\mathrm{J}$ Biogeogr 20(4):429-440

Merkle J, Fortin D, Morales J (2014) A memory-based foraging tactic reveals an adaptive mechanism for restricted space use. Ecology Letters 17(8):924-931

Moorcroft P, Barnett A (2008) Mechanistic home range models and resource selection analysis: a reconciliation and unification. Ecology 89:1112-1119

Moorcroft PR, Lewis MA (2006) Mechanistic home range analysis. Princeton University Press, Princeton

Othmer H, Dunbar S, Alt W (1988) Models of dispersal in biological systems. J Math Biol 26:263-298

Ovaskainen O (2008) Analytical and numerical tools for diffusionbased movement models. Theor Popul Biol 73(2):198-211

Ovaskainen O, Cornell SJ (2003) Biased movement at a boundary and conditional occupancy times for diffusion processes. J Appl Probab 40(3):557-580

Patlak C (1953) Random walk with persistence and external bias. Bull Math Biophys 15(3):311-338

Potts J, Bastille-Rousseau G, Murray D, Schaefer J, Lewis M (2014a) Predicting local and non-local effects of resources on animal space use using a mechanistic step-selection model. Methods Ecol Evol 5:253-262

Potts JR, Auger-Méthé M, Mokross K, Lewis MA (2014b) A generalized residual technique for analysing complex movement models using earth mover's distance. Methods Ecol Evol 5(10):10121022

Reeve JD, Cronin JT (2010) Edge behaviour in a minute parasitic wasp. J Anim Ecol 79(2):483-490

Reeve JD, Cronin JT, Haynes KJ (2008) Diffusion models for animals in complex landscapes: incorporating heterogeneity among substrates, individuals and edge behaviours. J Anim Ecol 77(5):898904

Rhodes J, McAlpine C, Lunney D, Possingham H (2005) A spatially explicit habitat selection model incorporating home range behavior. Ecology 86:1199-1205

Ries L, Fletcher RJ, Battin J, Sisk TD (2004) Ecological responses to habitat edges: mechanisms, models, and variability explained. Annu Rev Ecol Evol Syst 35:491-522

Salomon A, Waller N, McIlhagga C, Yung R, Walters C (2002) Modeling the trophic effects of marine protected area zoning policies: a case study. Aquat Ecol 36(1):85-95

Schultz CB, Franco AMA, Crone EE (2012) Response of butterflies to structural and resource boundaries. J Anim Ecol 81(3):724734

Thurfjell H, Ciuti S, Boyce M (2014) Applications of step-selection functions in ecology and conservation. Mov Ecol 2(1):4

Tjrve E (2010) How to resolve the \{SLOSS\} debate: lessons from species-diversity models. J Theor Biol 264(2):604-612

Turchin P (1991) Translating foraging movements in heterogeneous environments into the spatial distribution of foragers. Ecology 72(4):1253-1266

Turchin P (1998) Quantitative analysis of movement: measuring and modeling population redistribution in animals and plants, vol 1 . Sinauer Associates Sunderland, Massachusetts 
Tyson R, Thistlewood H, Judd GJR (2007) Modelling dispersal of sterile male codling moths, cydia pomonella, across orchard boundaries. Ecol Model 205(1-2):1-12

Xiao M, Reeve JD, Xu D (2013) Estimation of the diffusion rate and crossing probability for biased edge movement between two different types of habitat. J Math Biol 67(3):535-67
Zheng C, Pennanen J, Ovaskainen O (2009) Modelling dispersal with diffusion and habitat selection: analytical results for highly fragmented landscapes. Ecol Model 220(12):1495-1505

Zheng D, Chen J (2000) Edge effects in fragmented landscapes: a generic model for delineating area of edge influences (d-aei). Ecol Model 132(3):175-190 